\title{
Correspondence
}

\section{Comment on "Fisheries mismanagement"}

The recent article by O'Leary et al. (2011) raises an important question about the relationship between science and those who manage fisheries. They contend that fishery managers do not give due cognisance to scientific advice and consistently set Total Allowable Catches (TACs) above values advised by scientists (which they define as "political adjustment"). The authors claim that the consequence of this is that there is a high probability of stock collapse in the next 40 years. They use a simulation model to argue that this probability may exceed $80 \%$ at the mean level of political adjustment adopted by managers, depending on the degree of environmental variability and life history strategy of the fish. These claims are not supported by the analysis in the paper for the following reasons:

1. There are errors in the equations that describe the model.

2. The stochastic noise used to simulate recruitment variability is unrealistic.

3. The model is forced to produce high stock collapse rates without reference to real parameter values.

4. The life history characteristics of the model fish stocks are not representative of the fish species concerned.

5. The assumption that increasing the TAC always increases the out-turn catch is incorrect.

6. The assumption that juvenile fish are excluded from the TAC is incorrect.

In the appendix describing the model, Eqs. (A.3), (A.6), (A.8) and (A.9) are not correct as printed. If Eq. (A.4) represents what was actually done in the simulation it will produce zero or negative values for all spawning stock sizes which is clearly improbable yet is fundamental to the analysis.

The simulation model considers just one source of stochastic noise; that associated with recruitment. Unusually for a natural process the paper assumes a uniform error distribution with no mode where all events within a specified range are equally likely. In effect this means events at the extremes of the distribution are as likely to occur as those near the mean. Hence very poor recruitment is as likely as average recruitment, clearly giving a much greater chance of something bad happening to the stock than would be associated with a modal distribution more typically associated with natural populations. There is a wealth of literature on recruitment and its variability (see e.g. Myers, 2001) with good reason to suppose it has a clear mode and is right-skewed with a long tail giving lower probability to extreme events. Both the log-normal or gamma distributions have these characteristics

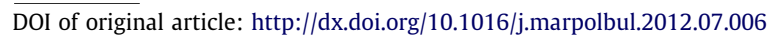

and are commonly used in simulation models (e.g. Kell et al., 2006).

However, there is a more serious question about the assumed underlying susceptibility to stock collapse. Unlike most models that are parameterised from data, the simulation model was forced in the "high environmental variability" case to produce a high probability of collapse: "High stochasticity was set according to the level of variation that led to a $10 \%$ chance of stock collapse within 20 years". This was done by manipulating of the amount of recruitment variability rather than allowing real estimates to determine the level of probability. A more realistic way to approach this would be to parameterise the recruitment error distributing from observations, of which there are a very large number (see Myers et al., 1995 for examples), to ensure that the probability distribution was related to real data. Given that the simulated stocks are putatively fished at Maximum Sustainable Yield (MSY), forcing a $10 \%$ chance of collapse appears arbitrary and means that in the 40 year experimental period in the simulation, collapse probability will rise to close to $20 \%$, even without TAC inflation, as can be seen in their Fig. 1a and c. One might reasonably ask why MSY would be considered a sustainable strategy with such high risks.

The authors argue that late maturing species ("cod-like") are less vulnerable to fishing than early maturing ("herring-like") species. This is contrary to experience as late maturing species often suffer high cumulative mortality up to the age of maturity making the probability of an individual reaching reproductive age very low. In fact the apparent difference reported in the paper has little to do with age of maturity but is the result of a very specific assumption about carrying capacity or "virgin biomass". The paper considers two recruitment strategies characterised by the "steepness" (Mace and Doonan, 1988) of the stock-recruitment function where the "late maturing" (cod-like) species has a low steepness (0.4) compared with 0.7 for the early maturing (herring-like). However, both species have the same virgin biomass (set to 100 ). This forces the low steepness case to have a higher $B_{\mathrm{msy}}$ which in turn means it is less likely to fished to collapse when fished at MSY. It also means that in the absence of fishing both species would have similar abundance and local equilibrium population dynamics. Hence all the simulation is doing to comparing the performance of two values of steepness, not full life history traits. The conclusion reached by the authors can be easily reversed by allowing virgin biomass to differ between species and hence capture more of the true life history qualities. Cod, for example, occupy a much higher trophic level than herring, and might be expected to be far less abundant. Historically North Sea cod reached maximum post-war spawning biomass of 250-300 thousand tonnes while the comparable figure for North Sea herring is an order of magnitude larger. It seems far more likely that the virgin biomasses of these species differ widely. Reducing virgin biomass for the cod-like stock by only a modest amount in the simulated populations would lead to the opposite conclusion. 
Furthermore, the choice of steepness appears arbitrary. Of $21 \mathrm{cod}$ stocks analysed by Rose et al. (2001) none had steepness as low as 0.4 and only 5 had steepness values less than 0.8 . High values tend to make a species more resilient to fishing. It is difficult to believe that the life history properties in the simulation reflect real populations of either cod or herring.

In one scenario the authors have assumed that the catch of juveniles is taken in addition to the TAC. There is an assumption that the TAC reflects mature fish alone. This is rarely the case, especially for late maturing species. In North Sea cod, for example the landings comprise a majority of immature fish and these are included in the TAC, as is the case for many other stocks. Hence it is not realistic to simulate the catch of juveniles separately from the TAC especially as there is no reason why immature fish cannot be a component of the MSY. The choice of mortality rate for juveniles is extremely high (50\%) and arises from an assumption that discard rates are mortality rates. Discard rates referred to in the paper (p. 2643) are the proportion of fish caught that are subsequently discarded, not the mortality rate. In North Sea cod, for example the discard rate at age 1 is over $90 \%$ but the exploitation rate (mortality due to fishing) is only about $20 \%$ because the proportion of fish caught at this age is low (ICES, 2011). Hence the selection of a $50 \%$ mortality rate is not an intermediate value as claimed but a high value. The combination of assuming the TAC only applies to the mature catch coupled with a high fixed additional mortality on juveniles will inevitably lead to higher collapse rates in the simulated population.

The effect of fishing is included in the simulation model as a harvest control rule based on the TAC being set to MSY depending on the stock biomass (Eq. (A.8)). It is argued that "in a well-managed fishery the catch target is set to maintain the stock at $B_{\mathrm{msy}}$ ". It is well known that uncertainties relating to assessment, modelling and implementation are such that a simplistic target of this type may well fail (see, for example, Cooke, 1999). For these reasons ICES harvest control rules are more widely tested to consider these uncertainties and are chosen on the basis of their robustness. The HCR used in the paper by O'Leary et al. does not reflect those used in the management of many stocks and may in any case be inherently risk prone, regardless of the actions of managers.

Overall the configuration of the simulation model and its parameterisation do not provide a reliable basis on which to quantify the probability of stock collapse. But there is a further twist to this problem which the authors have not considered. They have found that typically the TAC is set above the advised level. However, it is worth considering the actual out-turn catch that was achieved against the TAC. Table 1 shows the proportionate change of the realised catch from the TAC for the major stocks in the North Sea and West of Scotland. In almost all cases the realised catch was below the TAC and the mean shortfall is close the mean TAC inflation factor reported by O'Leary et al. This means that political adjustment of the TAC did not increase the catch as was assumed in the simulation.

It is unwise to blame managers and politicians for mis-management based on a poorly constructed model. There are deep-rooted socio-economic issues that undermine the performance of the current European management regime which need to be addressed in order for it to improve. Simple reliance on science alone is not a sufficient solution especially where the precision and accuracy
Table 1

Mean difference between the observed catch (Y) and the TAC expressed as a proportion of the TAC, for major stocks in the North Sea and West of Scotland for the period 1989-2010. Negative values mean the actual catch was less than the TAC.

\begin{tabular}{lll}
\hline Species & Stock & (Y-TAC)/TAC \\
\hline Cod & North Sea & -0.10 \\
Cod & West of Scotland & -0.25 \\
Haddock & North Sea & -0.24 \\
Haddock & West of Scotland & -0.36 \\
Whiting & West of Scotland & -0.38 \\
Saithe & North Sea and West of Scotland & -0.13 \\
Plaice & North Sea & -0.13 \\
Sole & North Sea & 0.09 \\
Herring & North Sea & 0.16 \\
Herring & West of Scotland & -0.35 \\
\hline
\end{tabular}

required for current the management process is barely achievable. When criticism of managers is justified it should be based on sound and comprehensive analysis.

\section{References}

Cooke, J.G., 1999. Improvement of fishery-management advice through simulation testing of harvest algorithms. ICES Journal of Marine Science 56, 797-810.

ICES, 2011. Report of the Working Group on the Assessment of Demersal Stocks in the North Sea and Skagerrak (WGNSSK), ICES CM 2011/ACOM13.

Kell, L.T., Pilling, G.M., Kirkwood, G.P., Pastoors, M.A., Mesnil, B., Korsbrekke, K., Abaunza, P., Aps, R., Biseau, A., Kunzlik, P., Needle, C.L., Roel, B.A., Ulrich-Rescan, C., 2006. An evaluation of multi-annual management strategies for ICES roundfish stocks. ICES Journal of Marine Science 63, 12-24.

Mace, P.M., Doonan, I.J., 1988. A generalised bioeconomic simulation model for fish population dynamics. New Zealand Fisheries Assessment Research Document $88 / 4,51 \mathrm{pp}$.

Myers, R.A., 2001. Stock and recruitment: generalizations about maximum reproductive rate, density dependence, and variability using meta-analytic approaches. ICES Journal of Marine Science 58, 937-951.

Myers, R.A., Bridson, J., Barrowman, N.J., 1995. Summary of worldwide spawner and recruitment data. Canadian Technical Report of Fisheries and Aquatic Sciences No. 2020, issue 2024. Northwest Atlantic Fisheries Centre, p. 327.

O’Leary, B.C., Smart, J.C.R., Neale, F.C., Hawkins, J.P., Newman, S., Milman, A.C., Roberts, C.M., 2011. Fisheries mismanagement. Marine Pollution Bulletin 62 2642-2648.

Rose, K.A., Cowan Jr., J.H., Winemiller, K.O., Myers, R.A., Hilborn, R., 2001 Compensatory density dependence in fish populations: importance, controversy, understanding and prognosis. Fish and Fisheries 2, 293-327.

Robin M. Cook

Department of Mathematics and Statistics, University of Strathclyde, Livingstone Tower, 26 Richmond Street, Glasgow G1 1XH, Scotland,

United Kingdom

Tel.: +44 (0)141548 3666; fax: +44 (0)141548 3345 . E-mail address: robin.cook@strath.ac.uk

Coby L. Needle

Marine Scotland Science, Marine Laboratory, 375 Victoria Road, Aberdeen AB11 9DB, Scotland, United Kingdom

Paul G. Fernandes Institute of Biological and Environmental Sciences, Zoology Building, University of Aberdeen, Tillydrone Avenue, Aberdeen AB24 2TZ, Scotland, United Kingdom 